$\xi=-$ 娄

\title{
The Design of Space Based on Architectural Geometry
}

\author{
Wasilah Wasilah* \\ Architectural Engineering, Faculty of Science and Technology Alauddin Islamic State University \\ *Corresponding author E-mail: wasilah@uin-alauddin.ac.id
}

\begin{abstract}
The aim of expressional space is to facilitate the society to indulge their hobby and talent autodidactically. The space for expression in Makassar City is necessary for the mural artist and the youngsters who have a hobby to play a skateboard. Their talent is not seriously attention from the government and creates negative attitude because there is no a space to accommodate their activity. This paper presents a design idea of expressional space with the implementation of the architectural geometry. The architectural geometry design principle is based on simple geometry form application as a realization of a form from the space. An experience of the space for the user realizes by the relationship between the space and the interaction with the environment through geometry and form processing as the basis of the creation process in the architectural works. Content analysis method providing a landscape layout and transformation of form and building mass in form of the implementation of architectural geometry principles. The principles are pure geometry, the composition of geometry, direction wall, and opening for the transformation process of expressional space form to produce and increases an aesthetic value of the environment.
\end{abstract}

Keywords: expressional space; geometry architecture; pure geometry; composition of geometry; mural art; skateboard; space form

\section{Introduction}

The definition of architecture based on the book of Hybrid Space is "The art or science of building; specify: the art or practice of designing structures and esp. inhabitable ones" [8]. This definition sharply defines the architecture as an art. An art definitely addresses to deliver something in aesthetic value. Kimberly Elam expresses "Architecture has some of the strongest educational ties to geometric organization because of the necessity for order and efficiency in construction, and the desire to create aesthetically pleasing structures" [3]. She explained the architecture has a strong relationship with a geometry. One of the connections between architecture and the geometry is aesthetic value. Therefore, obviously more services in architecture is popular nowadays, such as building architectural service, landscape architecture, the city architect, and etc.

According to the definition, the geometry is one of an element to present an aesthetic value in architectural works. In fact, in order to create the aesthetic value, then the architecture work should follow the geometry principles. This principle will define the result of the architecture form. One of the example in geometry principles is the golden section.

A roman architect, Marcus Vitruvius, explained the developer must always practice the precise ratio in the development of a temple, he stated "for without symmetry and proportion, no temple can have a regular plan" [7]. Every existing temple at that time must practice the golden section rule. As a result, the form of the most temple at that time are fairly similar and standard. According to the limitation of the golden section rule, there is a creative ideas limitation on the architects and architecture diversity at that time.

"The purpose of geometry of design is not to quantify aesthetics through geometry but rather to reveal visual relationships that have foundations in the essential qualities of life such as proportion and growth patterns as well as mathematics. Its purpose is to lend insight into the design process and give visual coherence to design through visual structure. It is through this insight that the artist or designer may find worth and value for themselves and their own work" [3]. Kimberly Elam description on the geometry function explained that the geometry has relevant function to express the visual relationship of an object based on its proportion and also the development pattern of the object.

The geometry function practiced in the architect on the building architecture. If the painting and the building has no geometry principles then the building consider does not have aesthetic value at that time. However, a lot of paintings and forms without geometry norm and the works may categorize as an aesthetic works. Obviously, the geometry rules in a design would limit the result of design variation. Other than the application of geometry as visualization relationship and a proportion of an object, geometry has a function as a norm to present a size in a building and shape.

Geometry is one of mathematics science that implemented in the architectural world and one of the branches of science that associated with size, composition, and proportion. One of a chapter in the essay of Dr. Joachil Langhein stated that "Proportion as a guiding pattern for establishing beauty." Moreover, the proportion has a close relationship with geometry, even non-geometry procedure that possibly presents a proportion. The proportion in a geometry related to symmetry that controls axis reflection, rotation, stretching, and etc. The geometry presents because of proportion and building symmetry. A geometry with symmetrical element provides a beauty in a building. According to Mangunwijaya definition, "Beauty is a matter of subjective. A culture of humanity will define the standard of a beauty 
based on their community (Dayak or Buginese ethnic group probably will have a different understanding in the definition of beauty), Their definition in beauty would provide by a generation to generation and create a specific 'beauty' mindset. A traditional building architectural aesthetic is one of the origins of the culture [6].

Mangunwijaya interprets a beauty is from a cultural, the traditional building. Additionally, more definition of a freedom of expression in the context of geometry is: Every people who follows design processing will present a different product, even they have an equal trigger. A diversity that exists in respective products is one of illustrative example in term of 'freedom of expression' [6]. This freedom refers to an understanding where the geometry does not limit a freedom of expression in architecture. This definition draws a conclusion where the geometry principle in a design understanding could restrict the result of a design variation. This understanding has changes along with the exploration of form, function, and various expression of architecture with the application of a geometry.

Finally, we will return to the basic of an architecture. Mangunwijaya in the book of Wastu Citra, writes about: "Berarsitektur or how to make an architecture is a language in terms of space and aspect or gatra, along with line and plane with a material and ambiance. We should create a proper architecture according to the culture, accompanying the feelings and responsibility in the application of a proper architectural language." A beauty in architecture is the architecture that aware on the value-in-use, following the aesthetic value as spiritual level inside the design. The result is not only a product that placed as "a statue", but the result is something that should provide an investigation on a meaning or three dimensions (or even the fourth dimension) [6]. Geometry never limits the expression in an aesthetic. In fact, geometry offers a freedom in the language of space, line, and plane.

Briefly, geometry would present a beauty in architectural art either in form of place or as an ornament and symbol. Geometry could represent an expression in a space. A difference in respective space function frequently explained with a different expression. In other words, the function would produce an expressional form. Generally, the expressional space is an accessible public area for all society. The public area is part of an environment completed with a pattern and function as a socialization and communication area between the society. Principally, the public area would define as a place to facilitate specific activity or interest for the people either individually or in the community.

The existences of expressional space have an influence on the economic development and social conflict that occur inside the youth because the expressional space becomes a facility to express their interest and individual skill. Examples of the activities in terms of the hobby are skateboard attraction and mural arts. Skateboard attraction and mural art are the activities that heavily consume more physical and thinking. These activities become a popular activity in the metropolitan city of Indonesia, includes the Makassar city.

According to this phenomenon, an idea hit upon to create an open space to facilitate the skateboard and mural community in Makassar city to limit the problems of street dysfunction and vandalism in public facility in some area of Makassar city street. The design of the public area expected would become a space for the skateboard community to express their hobby. Additionally, the mural community would have benefit, exhibit, and sells their artworks. Moreover, the result would bring an increase in a creative economy sector for the Makassar youngster. Furthermore, the aim of the design would welcome the existences of skateboard and mural community in Makassar city and these communities could socialize with the society. As an open space to facilitate the sport and art community, then the inspiration of space conceptual is architectural geometry concept that emphasizes the space aesthetic art.

The other side of geometry is the order geometry creation. People tend to produce a set of rules in their lives. Architecture is a facility to meet the needs of life and require the application of an order. The form creation cannot separate from the regular forms. The geometric shapes appear to reinforce the impression of space and creates an interior in geometry.

\section{Materials and Methods}

The research materials is site observation data about a potential condition and land suitability for arts and sports area. Then, the data processed by content analysis method [2]. Content analysis approach conducted in this study will realize the result of the design with architectural geometry application. The design methods of this study are:

1. Literature study: data collection on architectural geometry, expressional space, and space and standard requirement to facilitate the skateboard attraction and mural art.

2. Observing the site plan: collecting a series of activity information on skateboard and mural community, characteristic of the skateboard and mural community, characteristic of public area in Makassar city as a site plan to support a successful in the design of expressional space.

3. The analysis in design approach: identifying the architecture component of geometry [5], includes:

a) Geometry principles include an explicit composition of geometry form which is the pure geometry forms in terms of square, rectangle, circle, and triangle.

b) The composition of geometry: A shape of geometry is not absolute. Therefore, the form is part of the transformation process, a form transformation may understand as a result of variance from existing basic forms manipulation.

c) Direction wall consist of wall to cover a space and building. Sometimes the wall may refer to a building envelope. The wall is one of important elements to present a form of a building. The wall visual is dominant rather than the element of floor and roof plane as part of the building envelope.

d) The opening. There is a close relationship between the opening and the wall. The visual space quality certainly determines by the opening. For example, the wall opening organization would produce a reflection of illuminance. Generally, the opening will frame and receive the landscape view enjoyment from the interior area, as part of indoor and outdoor confrontation [4]. The opening may identify as a large opening. The large opening is a result of size reduction form or vertical opening of the building mass volume and also an opening from the interval between every wall surface.

4. Concept formulation, applying the configuration of expressional space with the implementation of the architectural geometry principles that integrated with a site plan environmental aspect. Architectural geometry principle emphasizes building aesthetic and highquality of the environment.

5. The result of the design is in form of expressional space draft with the application of architectural geometry that presenting an expressive and innovative model for the public area. 


\section{Results}

\subsection{Landscape layout transformation based on architectural geometry principles}

\subsubsection{Pure geometry}

The site pattern created from the combination of pure geometry shapes, such as square, rectangle, circle, and triangle. Each pure geometry shape will synchronize with the function of the facilitated activity. The square shape representing the supporting facility area and mural area. The rectangle shape representing as a skateboard attraction area, field, Bosowa sport centre, and management office. The circle represents a rounded plants area and fountain spot. The triangle represents the triangular park area, parking area, and mural area. The shape of pure geometry organizes carefully to create and improve the aesthetic value of the site plan. The combination result of pure geometry shapes, such as square rectangle, circle, and triangle, described in Figure 1.

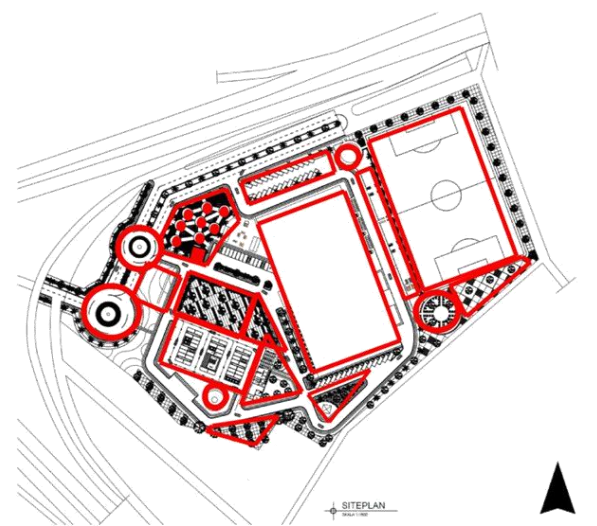

Fig 1: Combination of pure geometry shapes, square, rectangle, circle, and triangle are a basic of site plan layout pattern formation for expressional space

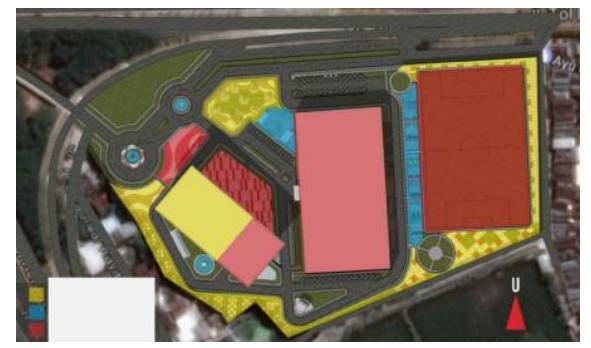

Fig 2: Allocation of space function based on activity types of the area.

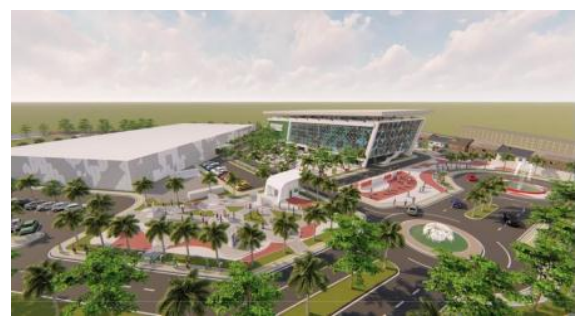

Fig 3: Realization of expressional space with the application of pure geometry shapes element.

\subsubsection{Geometry composition}

As described in Figure 1 and Figure 2, there is a form transformation as a result variances of existing basic shapes manipulation, such as the combination of rectangle and triangle shape and the combination of square and triangle shape. The processes of basic form manipulation are:

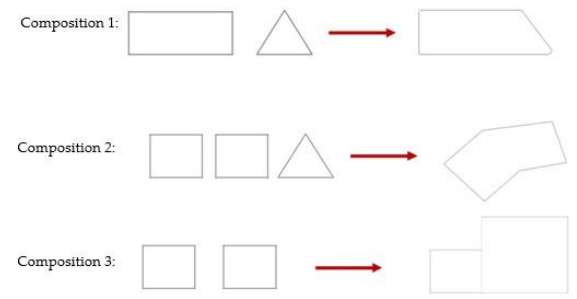

There is a complex form composition in the site plan design in the mural area at the front of the entrance. The composition shapes are 
square, triangle, circle, and rectangle shape that creates a pure geometry form. In addition, there is a separate part of pure geometry form, but the form has been transformed.

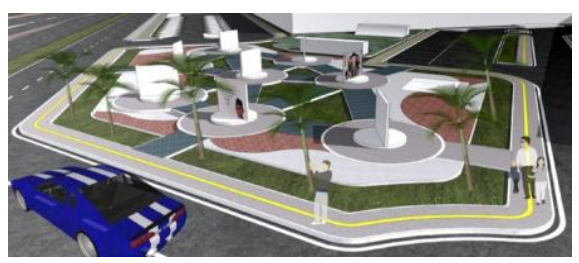

Fig 4: Mural area perspective

\subsection{Transformation of form and building mass based on the architectural geometry principles}

\subsubsection{Pure geometry}

There are mass buildings in the site plan design, a sport center and skateboard area. These areas supporting with infrastructures, such as management office, cafeteria, mushallah, and other infrastructures. The building shapes consists of rectangle and circle as the basic form, based on the skateboard formation which distorted into the main building form idea in the site plan design. Additionally, the main building facade has geometry pattern implementation to strengthen the geometry concept as a design approach concentration. Geometry pattern in the facades are consist of a circle, triangle, and square as the basic forms.

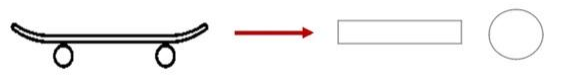

Fig 5: The skateboard basic form consists of rectangle and circle forms.

\subsubsection{Geometry composition}

The building formation is similar to a rigid box of rectangle completed with a massive wall that covers the entire building. The transformation of rectangle and circle pure geometry form gradually has a transformation even categorizes as a simple transformation form. The form is a result of metaphor process from a skateboard form. Moreover, the final result of the building design adapted the traditional house of Makassar Bugis tribe. According to the structure, the vertical building consists of foot, body, and the head of the building. As a result, the vertical main building consists of three main parts as similar with the traditional house building of Bugis, with three structures (foot, body, and head of building).

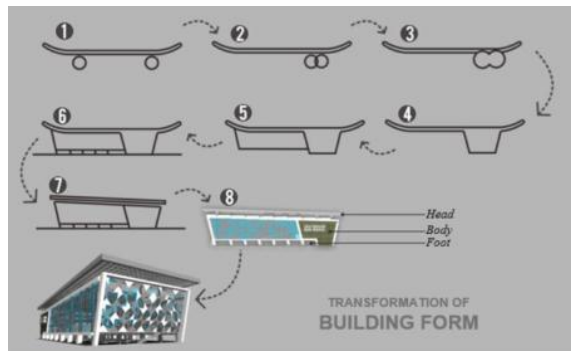

Fig 6: Transformation of building form

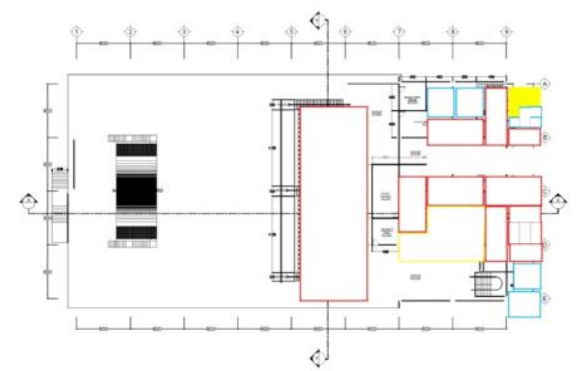

Fig 7: Main building ground plan on the second floor. Colour: Red (rectangle shape), Blue (square shape), Yellow (composition 3), Grey (building corridor).

According to the building ground plan, the square and rectangle structure is an intersection. The free-standing wall of the building is the intersection from one space to another space and create the interlocking entrance area. The free-standing wall positioning with similar pattern creates an interior room with an opening in the wall as illuminance area receiver. The geometry composition is a simple transformation form but the composition creates a visual interaction inside and outside of the building.

\subsubsection{Direction wall}

Basically, the function of the wall is the cover of a space and building, and the part of building envelope. According to Tadao Ando, 
walls are the most basic elements of architecture, a border between the interior and exterior, but at the same time wall can also be the most enriching element that would transform the form of a place and create a new form or new space [1].

Wall is an important element to present a form of a building because the wall is visually dominant rather than the ground plane and the roof plane, which are also the building envelope element. The floor is not directly visualized because the floor area is hidden by the wall and the roof plane usually a flat cover plane. The sport centre main building with dynamic wall positioning creates and divided the space. The wall function is a direction wall of a movement or user circulation line of the building. Although, the circulation track impression is fairly simple other than the building entrance towards to other rooms inside the building, in form of straight line.

The sport centre building exterior wall includes and covers most of the part of the site. The building contains the glass material to support the visitor enjoy two atmospheres simultaneously, the indoor with modern vintage ambiance and a fascinating outdoor view (a collaboration between mural art and the park). The glass material is from geometry pattern with triangle and square form combination.

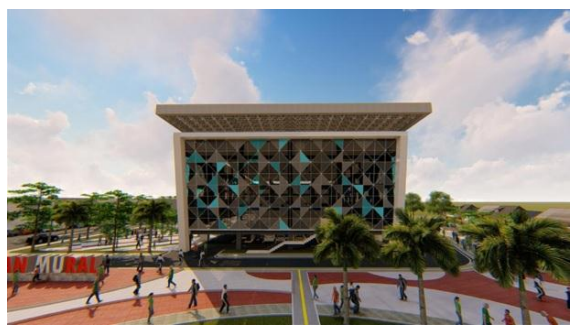

Fig 9: Geometry pattern in the exterior wall

\subsubsection{Opening}

The opening and wall in the sport centre main building design are two closely interrelated parts because the visualization room quality exactly determined by the opening. The opening becomes the part that organizes on the wall panel to create illumination possibility in natural ways. Most of the opening is to frame and receive the landscape view in order to enjoy from the interior room and also as an indoor and outdoor confrontation. Indoor expressional space is an opening in form of a vertical and large opening in building mass volume and an opening from the interval of the wall surface.

The third floor has an opening in the wall panel continuously to provide a natural illumination inside the building and improve the quality of space. These openings are located on every side of the building. This is one of the effort to gain a benefit of landscape condition around the building as the supporting aesthetic element from inside the building. In addition, the second floor has a touch of glass material opening to include the illumination element as a lighting in the transition area from inside into the outside or vertical opening. The second-floor opening will frame and present a vista, such as park and mural art display as a technique to combine the inside and outside of the vertical opening. A patterned-glass material creates a reflection with geometry forms.

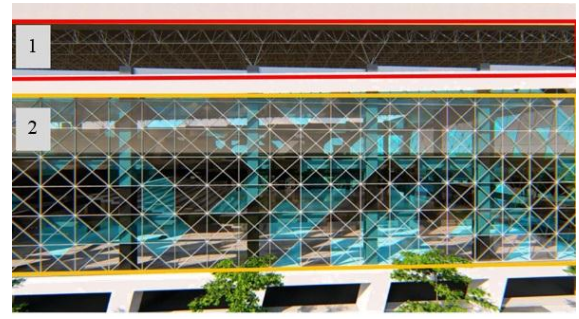

Fig 10: Opening in the main building. Number : 1 (Opening in the third floor), 2 (Opening of the second-floor building with a patterned-glass material)

\section{Discussion}

The site pattern design practiced the basic principles of geometric architecture. The implementation is gradually from the shape of pure geometry covering triangle, square, rectangle, and circle. The basic form transformed into a new pattern and the transformation process is categorizes as a simple transformation form.

Basically, the site plan area has three functional area categories, skateboard attraction area, mural area, and infrastructure for skateboard and mural areas. Skateboard attraction area indicated by a blue color in Figure 2 and located in two different areas, both side of the main building of Bosowa sport centre. The mural works exhibition display is located in the main street of site plan area. A decision of skateboard attraction area location is based on the accessibility reason, creating an accessibility for the visitor. Consequently, the skateboard area is located in the parking area as the strategic area. As well as the mural area, the area is located in two different areas which are along the site border in form of a high wall. The wall functioned as mural media and another area is located right in the entry point of the site. As a result, the visitor who enters the site will directly experience a mural art atmosphere that emphasizing the architectural geometry principles in expressing an aesthetic value.

In the main building, geometric shape visible on the building's facade with a patterned-glass material. The pattern comes from triangular and square shapes with a color degradation aims to produce shadow geometric pattern which dynamic in the direction movement of the sun shadow. While the covering wall of space in the building is simple with a grid pattern formed from the arrangement of a square and rectangular shapes to maximize the use of space for various activities. 
The design should be create a balance between complex form composition of art mural display and simple spatial layout patterns. Although the geometry form is applied quite simple, but the application of architectural geometry principle emphasizes building aesthetic and high-quality of the environment.

\section{Conclusions}

Geometry form would generate an aesthetic architecture art, either in form of space or as an ornament. Geometry could represent an expression in a space. Geometry will produce an expressive form. The geometry design principles -in form of pure geometry, a composition of geometry, direction wall and opening- are a representative form that explained in in expressional space design. The design idea is an architecture works that combine basic form variations, square, circle, rectangle, and triangle. These forms carefully organize to create and improve the aesthetic value of the area. The presentation of geometry form is fairly simple but have a variation. As a result, the creation of circulation pathway is simple. The circulation track creates by the direction wall. The application of simple geometry form is an effort to maximize the space as an area for skateboard attraction activity inside the building. Moreover, most of the opening building part is vertical opening with a standard of building user eye level. Patterned-glass material opening produces a harmonic geometry shadow formation.

\section{Acknowledgments}

Geometry will always exist in architecture in a variety of building angles. Either from the application of the floor plan, the building facade, in two dimensional or three dimensional is not separated from the geometric shape, judging from the elements of the whole. The shape of this form may affect design in the architectural concept. The other side of geometry is the order the creation. Humans tend to produce a set of rules in their lives. Architecture that is a facility to meet the needs of life is also required to apply for an order. Therefore, the forms creation cannot separate from the regular forms.

Authors would like to show our gratitude to the Architectural Engineering laboratory staff, Faculty of Science and Technology Alauddin Islamic State University, for sharing their pearls of wisdom with us during the course of this research.

\section{Reference}

[1] Ando, Tadao. 2008. Tadao Ando 3 Inside Japan. Japan: Nobuyuki Endo.

[2] Ekomadyo, A. S. 2006. Prospek Penerapan Metode Analisis Isi (Content Analysis) dalam Penelitian Media Arsitektur [Prospect in Implementation of Content Analysis Method in Research on Architectural Media]. Jurnal Ilmu Pengetahuan Teknologi dan Seni, Volume 10 (2), 51 -57.

[3] Elam, Kimberly. 2001. Geometry of design: Studies in Proportion ang composition. New York: Princeton Architectural Press.

[4] Gill, S. S. 2010. A Study of The Characteristics of Natural light in Selected Buildings Designed by Le Corbusier, Louis I. Kahn and Tadao Ando (Doctoral dissertation, Texas A \& M University).

[5] Hassan, Soraya Masthura. 2017. Prinsip Desain Geometri Arsitektur [Design Principles in Architectural Geometry], Tadao Ando. EMARA Indonesian Journal of Architecture, Volume 3 Nomor 2.

[6] Mangunwijaya, Y.B. 2009. Wastu Citra. Jakarta: PT. Gramedia Pustaka Utama.

[7] Vitruvius. 2014. The Architecture of Marcus Vitruvius Pollio. Createspace Independent Publishing Platform.

[8] Zellner, Peter. 1999. Hybrid Space: New Forms in Digital Architecture. London: Thames \& Hudson.

\section{Appendix A}

\section{A.1. Dimensions of the built space}

In this appendix, we present the collecting data about dimensions of the built space used for optimization of spatial distribution based on the contains activity proportion.

Table 1: The size of build space

\begin{tabular}{|l|l|l|}
\hline No & Space Name & Size (m2) \\
\hline 1 & Ground Floor (Parking and Service Area) & 466,57 \\
\hline 2 & 1st Floor (Skateboard Arena and Management Office) & 570,05 \\
\hline 3 & 2nd floor (Cafeteria and Mushallah) & 434,33 \\
\hline 4 & Security Room & 33,8 \\
\hline 6 & Site plan & 3811,08 \\
\hline & Total of built space & $\mathbf{5 3 1 5 , 8 3}$ \\
\hline
\end{tabular}

Based on the data obtained the amount of space utilization planning area are:

- Built space of 5315,83 $\mathrm{m}^{2}$

- Planning footprint area of $39122,51 \mathrm{~m} 2$

- Percentage of constructed land is: $5315,83 \mathrm{~m}^{2} / 39122,51 \mathrm{~m} 2 \times 100=13,587 \mathrm{~m}^{2}$ or $14 \%$

- Open space of 33,806 $\mathrm{m}^{2}$

- Percentage of open space : $33,806 \mathrm{~m}^{2} / 39122,51 \mathrm{~m}^{2}$ x $100=86,410 \mathrm{~m}^{2}$ or $86 \%$

The percentage of master plan in expressional space planning for skateboard and mural communities in Makassar city is $86 \%$ to support the main functional area, and $14 \%$ as supporting facilities within the planning area. 